\title{
Effect of Patient Arm Motion in Whole-Body PET/CT
}

\author{
Martin A. Lodge ${ }^{1}$, Joyce C. Mhlanga ${ }^{1}$, Steve Y. Cho ${ }^{1,2}$, and Richard L. Wahl ${ }^{1,2}$ \\ ${ }^{I}$ Division of Nuclear Medicine, Russell H. Morgan Department of Radiology and Radiological Sciences, Johns Hopkins University \\ School of Medicine, Baltimore, Maryland; and ${ }^{2}$ Sidney Kimmel Comprehensive Cancer Center at Johns Hopkins, Johns Hopkins \\ University School of Medicine, Baltimore, Maryland
}

\begin{abstract}
Arm motion during whole-body PET/CT acquisition is not uncommon and can give rise to striking cold artifacts on PET images. We investigated the mechanisms that underlie these artifacts and proposed a potential solution. Methods: A phantom experiment based on 5 clinical cases of suspected arm motion was designed. The experiment involved a central 20$\mathrm{cm}$-diameter ${ }^{68} \mathrm{Ge} /{ }^{68} \mathrm{Ga}$ cylinder simulating the neck and 2 peripheral $10-\mathrm{cm}$-diameter ${ }^{18} \mathrm{~F}$ cylinders simulating arms. After motion-free CT and PET on a whole-body PET/CT system, the position of the arms was altered so as to introduce different amounts of misalignment. Twenty sequential PET scans were acquired in this position, alternating between 2-dimensional (2D) and 3-dimensional (3D) acquisition, as the ${ }^{18} \mathrm{~F}$ decayed. Decay of ${ }^{18} \mathrm{~F}$ in the arms, while the activity in the ${ }^{68} \mathrm{Ge} /{ }^{68} \mathrm{Ga}$ cylinder remained approximately constant, allowed the relative impact of scatter and attenuation-correction errors to be determined. Results: Image artifacts were largely confined to the local region of motion in $2 \mathrm{D}$ but extended throughout the affected slices in 3D, where they manifested as a striking underestimation of radiotracer concentration that became more significant with increasing misalignment. For 3D, scattercorrection error depended on activity in the arms, but for typical activity concentrations scatter-correction error was more significant than attenuation-correction error. 3D image reconstruction without scatter correction substantially eliminated these artifacts in both phantom and patient images. Conclusion: Reconstruction artifacts due to patient arm motion can be substantial and should be recognized because they can affect both qualitative and quantitative assessment of PET.
\end{abstract}

Key Words: PET/CT; artifact; motion; arm; scatter correction

J Nucl Med 2011; 52:1891-1897

DOI: 10.2967/jnumed.111.093583

$\mathbf{F}$ or many clinical PET/CT studies, imaging of the full extent of the extremities is not essential and whole-body acquisition is commonly performed with the patient's arms raised above the head $(1,2)$. This positioning has the advantage of reducing photon attenuation in the region below the

\footnotetext{
Received May 23, 2011; revision accepted Aug. 17, 2011.

For correspondence or reprints contact: Martin Lodge, Johns Hopkins Hospital, Radiology/Nuclear Medicine Department, 600 N. Wolfe St., Baltimore, MD 21287.

E-mail: mlodge1@jhmi.edu

Published online Nov. 11, 2011.

COPYRIGHT @ 2011 by the Society of Nuclear Medicine, Inc.
}

shoulders, which represents most of the target scan range for typical whole-body PET/CT studies. For CT, this positioning decreases the incidence of beam-hardening artifacts (3), and for PET, it increases the total number of detected coincidence events and improves image statistical quality. In addition, having the arms raised, as opposed to having them by the patient's side, reduces the likelihood of truncation artifacts $(4,5)$.

Maintaining the arms in a raised position can be uncomfortable for many patients, and in our experience, arm motion during the imaging procedure is not uncommon. Although the distal arms may be outside the imaging field of view, the shoulders and proximal arms typically would be included. Because of the sequential nature of PET/CT acquisition, arm motion typically gives rise to spatial misalignment between the CT and PET data. Misaligned CT and PET images can complicate localization of tracer uptake, but a potentially more significant problem can arise because of a breakdown in the assumptions required for PET image reconstruction. Because PET relies on accurately aligned CT for both attenuation and scatter correction, patient arm motion can cause substantial artifacts in PET images reconstructed using misaligned $\mathrm{CT}$ data.

Although the effect of arm motion during PET/CT acquisition has not been specifically addressed to date, other motion issues have been described in detail: respiratory motion in oncology $(6,7)$ and cardiology $(8,9)$, gross patient movement in cardiology $(10,11)$ and neurology $(12,13)$, and internal organ motion $(14,15)$. In the present study, we investigate the mechanisms that underlie the sometimes profound artifacts that can result when patient motion causes CT and PET images of the arms to become misaligned.

\section{MATERIALS AND METHODS}

A phantom study was performed to assess the impact of arm motion during PET/CT data acquisition. The design of this phantom study was guided by clinical cases involving suspected arm motion that were observed in our PET/CT practice. An institutional review board exemption allowed review of our PET/ CT image database. Five patient studies were identified during clinical reading sessions as having unusual cold artifacts and suspected misalignment of the arms. All data were acquired using a Discovery VCT (RX) (GE Healthcare) clinical whole-body PET/ CT system (16). According to standard protocols, patients were positioned supine with arms raised and supported above the head. 
CT acquisition (64-slice; pitch, 0.984; $120 \mathrm{kVp}$; 20-200 mA; 0.5-s rotation time) from the base of the skull to mid thigh was immediately followed by multibed PET acquisition (lutetium yttrium orthosilicate crystals; 3-dimensional [3D] acquisition; $255 \mathrm{~s}$ per bed position; 7 bed positions; $36-\mathrm{mm}$ bed overlap) in the caudocranial direction. Three different PET image series were reconstructed using ordered-subsets expectation maximization (2 iterations; 21 subsets; 3 mm gaussian filter; 4.69-mm pixels; $3.27-\mathrm{mm}$ slice thickness) and the following processing combinations: no attenuation correction and no scatter correction (NAC-NSC), CT-based attenuation correction (17) and model-based scatter correction (18) (AC-SC), and CT-based attenuation correction and no scatter correction (AC-NSC).

On the basis of these clinical data, a phantom experiment was designed consisting of a 20 -cm-diameter ${ }^{68} \mathrm{Ge} /{ }^{68} \mathrm{Ga}$ resin cylinder (Sanders Medical Products) simulating the neck and two $10-\mathrm{cm}-$ diameter water-filled cylinders simulating arms in the raised position. The ${ }^{68} \mathrm{Ge} /{ }^{68} \mathrm{Ga}$ cylinder was oriented parallel to the $z$-axis of the scanner and centered within the transverse field of view. The 2 smaller cylinders were positioned laterally on either side of the ${ }^{68} \mathrm{Ge} /{ }^{68} \mathrm{Ga}$ cylinder and were initially oriented parallel to the $z$-axis. The smaller cylinders were filled with ${ }^{18} \mathrm{~F}$ so as to have an activity concentration at the start of the first PET acquisition (10.7 $\mathrm{kBq} / \mathrm{mL})$ that was similar to the ${ }^{68} \mathrm{Ge} /{ }^{68} \mathrm{Ga}$ cylinder $(10.9 \mathrm{kBq} / \mathrm{mL})$. The intention of using ${ }^{18} \mathrm{~F}$ was to introduce different amounts of activity in the arms over the course of an experimental period that lasted over ten ${ }^{18} \mathrm{~F}$ half-lives. This time period allowed for essentially complete decay of the ${ }^{18} \mathrm{~F}$ but negligible decay of the ${ }^{68} \mathrm{Ge} /{ }^{68} \mathrm{Ga}$.

CT data were acquired using the parameters described above, over a $15-\mathrm{cm}$ axial range that matched a single PET field of view. PET data were then acquired over the same range, first using 3D mode $(9 \mathrm{~min})$ and then a separate acquisition in 2-dimensional (2D) mode (18 min). After completion of these scans, in which the CT and PET were perfectly aligned, the phantom arrangement was adjusted to simulate arm motion. The positions of the two ${ }^{18} \mathrm{~F}$ cylinders were altered by moving one end of each cylinder in the lateral direction, $5 \mathrm{~cm}$ away from its original location. Whereas originally these 2 outer cylinders were parallel to the $z$-axis of the scanner, after translation they were oriented at approximately $18^{\circ}$ with respect to this axis. The effect of this motion was to introduce a degree of misalignment between the

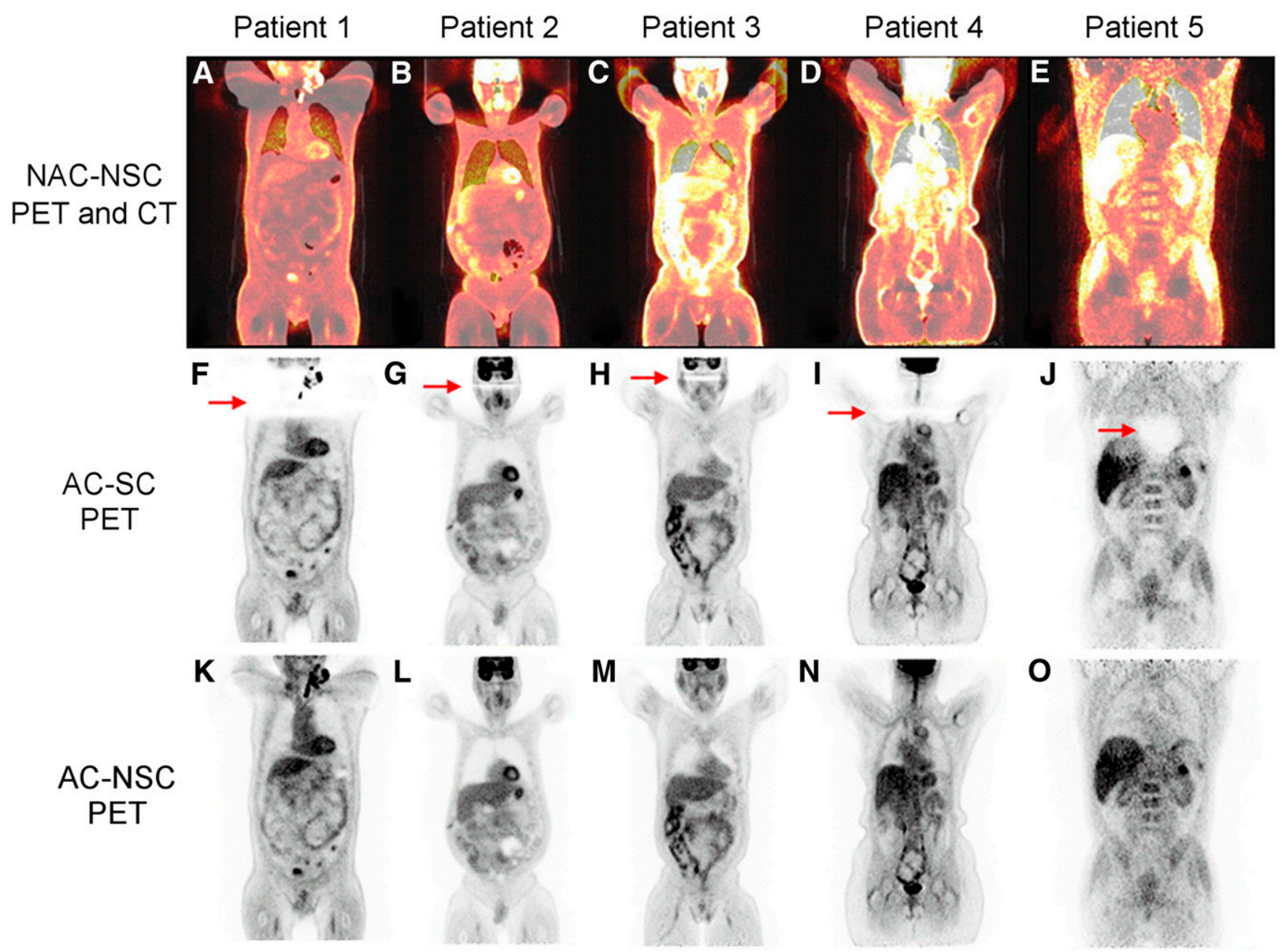

FIGURE 1. Five different patient examples showing artifacts in coronal PET images consistent with arm motion. Each column shows data from different patient. First 4 columns show PET data obtained with ${ }^{18} \mathrm{~F}-\mathrm{FDG}$ (median, $61 \pm 16$ min of uptake and $\left.635 \pm 67 \mathrm{MBq}\right)$. Last column (E, J, and O) shows PET data obtained with ${ }^{11} \mathrm{C}$-acetate (28 min of uptake and $639 \mathrm{MBq}$ ). (A-E) NAC-NSC PET, displayed in hot-body color scale, superimposed on CT. CT data are displayed in lung window to emphasize body outline and highlight arm motion that occurred between acquisition of CT and of PET. (F-J) Fully corrected AC-SC PET images with cold artifacts evident at level of arm motion, indicated by red arrows. (K-O) AC-NSC PET images in which cold artifacts were substantially reduced by not applying scatter correction. All PET data were acquired in 3D mode. 
position of the outer cylinders and their previous position at the time of the CT. The extent of this misalignment varied with axial position and ranged from 0 to $5 \mathrm{~cm}$. With the phantom in this new position, a further 18 sequential PET scans were acquired, alternating between $3 \mathrm{D}(9 \mathrm{~min})$ and $2 \mathrm{D}(18 \mathrm{~min})$ modes, over a period of $270 \mathrm{~min}$. An additional pair of 3D and 2D images was also acquired after a delay of $20 \mathrm{~h}$ after the start of the first PET acquisition. The initial scans performed before phantom motion were labeled 3D0 and 2D0, and the subsequent scans performed after phantom motion were labeled 3D1-3D10 and 2D1-2D10.

PET images were reconstructed using either 2D or 3D orderedsubsets expectation maximization as appropriate, in conjunction with the parameters described for the clinical protocol. The same 3 correction combinations (NAC-NSC, AC-SC, and AC-NSC) were also applied. When scatter correction was used, the method of Bergström et al. (19) and a model-based approach (18) were used for 2 and 3 dimensions, respectively. Quantitative image analysis used $19-\mathrm{cm}$ circular regions of interest manually positioned within the ${ }^{68} \mathrm{Ge} /{ }^{68} \mathrm{Ga}$ cylinder for all images slice, excluding the extreme end slices.

\section{RESULTS}

Figure 1 shows coronal images from 5 patient studies that illustrate the artifacts associated with arm motion. In Figures $1 \mathrm{~A}-1 \mathrm{E}$, the extent of the arm motion can be visualized as regions of spatial misalignment between the $\mathrm{CT}$ images and the NAC-NSC PET images, which are independent of the CT data and reflect the real position of the arms at the time of the PET acquisition. In Figures $1 \mathrm{~F}-1 \mathrm{~J}$, red arrows indicate localized regions of apparently low tracer concentration in the conventional AC-SC images. These cold regions are suspi- cious because they appear as sharp discontinuities that are not consistent with the expected distribution of the tracer. In each case, the apparent artifacts arise at the same axial level as the arm motion and occur in multiple transverse slices depending on the extent of the motion. The artifact is not localized to within the arm or shoulder region where PET and CT misalignment occurred and typically extends throughout the affected transverse slices. Although 4 of the 5 cases presented here involved ${ }^{18} \mathrm{~F}$-FDG, the artifact does not appear to be related to the PET radiopharmaceutical because the artifact also occurred in a separate study performed with ${ }^{11}$ C-acetate (Fig. 1J).

Figures 2 and 3 show representative coronal slices from the phantom study, acquired in $3 \mathrm{D}$ and $2 \mathrm{D}$ modes, respectively. The first column (A, F, and K) shows images acquired with perfect alignment of the PET and CT data. Subsequent columns show PET images in which the 2 outer cylinders had been moved with respect to the CT to simulate arm motion. The top rows (A-E) show NAC-NSC PET images superimposed on the CT images and clearly show the misalignment in B-D. The middle rows (F-J) show fully corrected (AC-SC) PET images. Inspection of the PET images from left to right shows decreasing activity in the arms due to radioactive decay of ${ }^{18} \mathrm{~F}$ and negligible arm activity in the last column. The blue arrows in both the $3 \mathrm{D}$ and the $2 \mathrm{D}$ images (Figs. 2 and 3) indicate artifacts in the arm regions that are consistent with inaccurate attenuation correction due to misalignment of the CT and PET data. The red arrows in the 3D data (Fig. 2) indicate a separate artifact that manifests

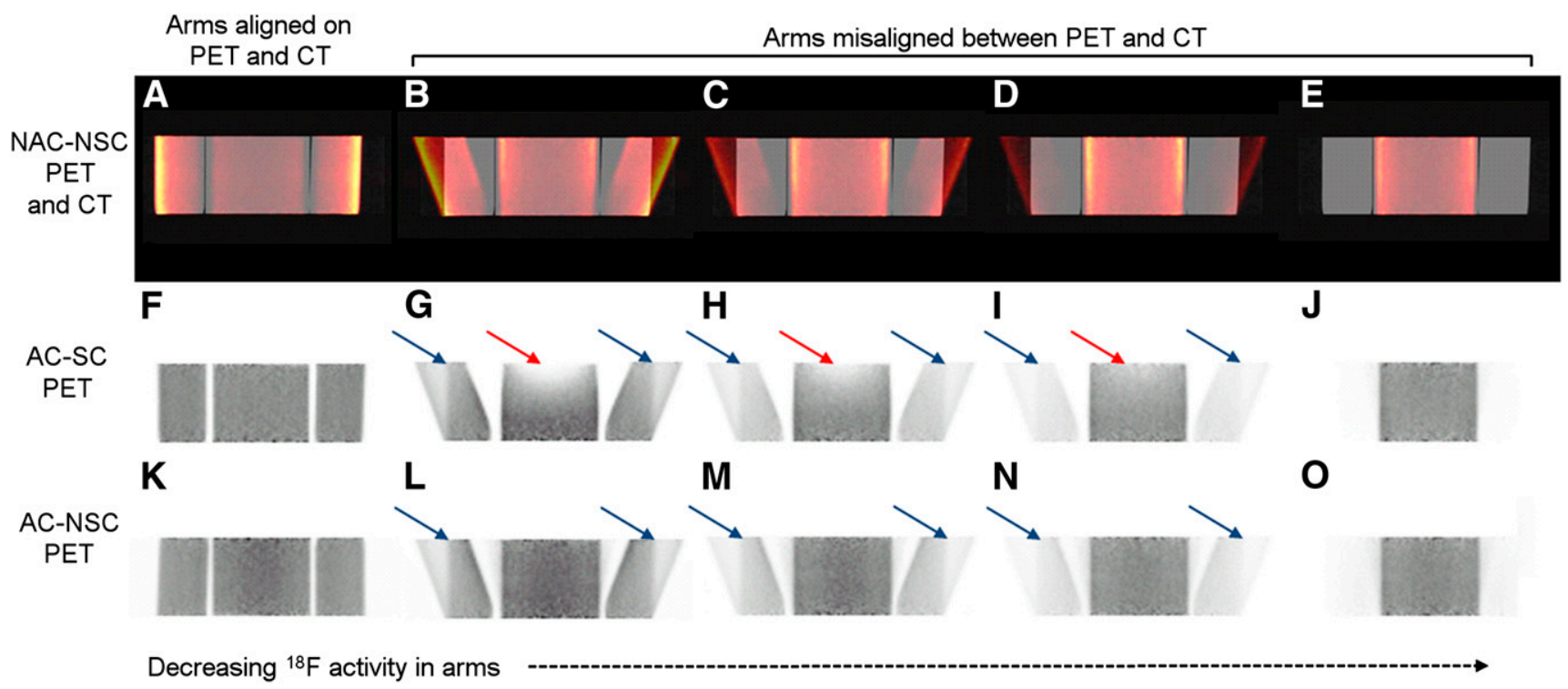

FIGURE 2. Coronal images of phantom arrangement acquired in 3D mode. First column represents 3D0 and was acquired with perfect alignment of PET and CT. Subsequent columns represent 3D1, 3D4, 3D7, and 3D10, respectively, and were acquired with PET and CT misaligned. Central ${ }^{68} \mathrm{Ge} / 68 \mathrm{Ga}$ cylinder was stationary, whereas 2 outer ${ }^{18} \mathrm{~F}$ cylinders were intentionally misaligned with respect to $\mathrm{CT}$ to simulate arm motion. ${ }^{18} \mathrm{~F}$ activity is seen to decay from left to right and was negligible at time that images in last column (E, J, and $\mathrm{O}$ ) were acquired. (A-E) NAC-NSC PET, displayed in hot-body color scale, superimposed on CT. CT data are displayed in lung window to emphasize phantom outline and highlight motion that occurred between acquisition of CT and PET (except first column). (F-J) Fully corrected AC-SC PET images, with blue arrows indicating artifacts in vicinity of arm motion. Red arrows indicate other, more centrally located, cold artifacts. (K-O) AC-NSC PET images, in which central cold artifacts were substantially reduced by lack of scatter correction. 


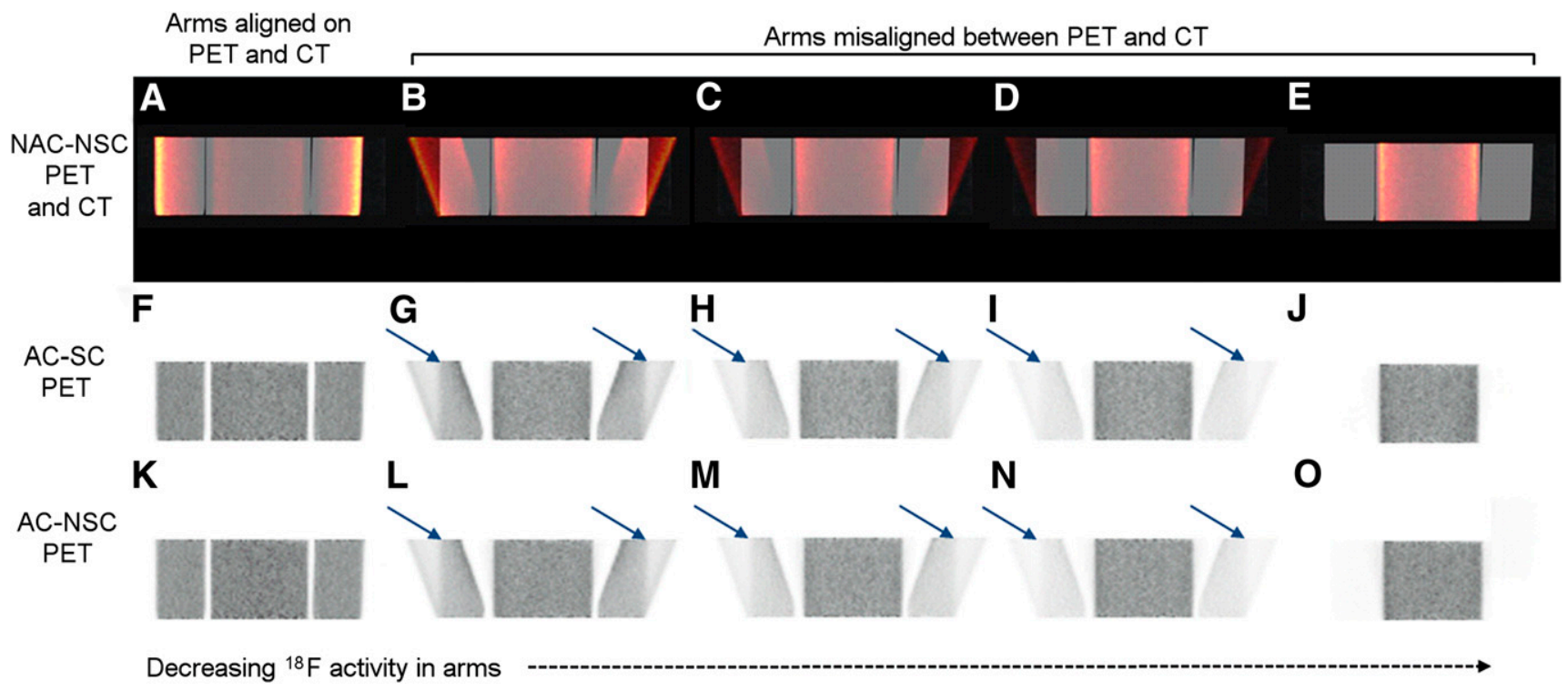

FIGURE 3. Coronal images of phantom arrangement acquired in 2D mode. First column represents $2 \mathrm{D} 0$ and was acquired with perfect alignment of PET and CT. Subsequent columns represent 2D1, 2D4, 2D7, and 2D10 and were acquired with PET and CT misaligned. Central ${ }^{68} \mathrm{Ge} /{ }^{68} \mathrm{Ga}$ cylinder was stationary, whereas 2 outer ${ }^{18} \mathrm{~F}$ cylinders were intentionally misaligned with respect to CT to simulate arm motion. ${ }^{18} \mathrm{~F}$ activity is seen to decay from left to right and was negligible at time that images in last column (E, J, and O) were acquired. (A-E) NACNSC PET, displayed in a hot-body color scale, superimposed on CT. CT data are displayed in lung window to emphasize phantom outline and highlight motion that occurred between acquisition of CT and PET (except first column). (F-J) Fully corrected AC-SC PET images, with blue arrows indicating artifacts in vicinity of arm motion. (K-O) AC-NSC PET images, which, in case of these 2D data, are qualitatively similar to AC-SC images shown in F-J.

as a cold region, more centrally located and some distance from the site of arm misalignment. This artifact becomes increasingly more pronounced as arm misalignment increases, as can be seen on moving from the bottom to the top of these coronal images. The artifact is also seen to be most pronounced when activity in the arms was greatest $(\mathrm{G})$ and declines with decaying ${ }^{18} \mathrm{~F}$ activity such that there is no obvious artifact when there is no activity in the arms (J). This central cold artifact is seen only in the 3D images and is not evident in the $2 \mathrm{D}$ images, despite the fact that the phantom configurations were identical in each case.

Scatter fractions estimated during scatter correction of the AC-SC images are shown in Table 1 for the entire axial field of view. The scatter fraction is substantially higher for 3D1 than for the correctly aligned 3D0 and decreases with each subsequent scan. This decrease in the 3D scatter fraction between 3D1 and 3D10 occurs despite the fact that the phantom geometry is unchanged. The only difference between these phantom arrangements is the decreasing ${ }^{18} \mathrm{~F}$ activity in the misaligned arms. The scatter fraction for 3D10 $(0.30)$, which had no arm activity, is approximately the same as 3D0 (0.31), suggesting that it is the presence of radioactivity in the misaligned arms (as opposed to a real change in the scatter distribution) that is responsible for overestimation of the scatter fraction. These data suggest that overcorrection for scatter may contribute to the central cold artifacts seen in the 3D AC-SC images. PET images reconstructed with AC-NSC are shown in Figures 2 and $3(\mathrm{~K}-\mathrm{O})$. The 3D AC-NSC images show a substantial reduction in the central cold artifact as a result of the absence of scatter correction.
Figure 4 quantifies the extent of the central cold artifact as a function of image slice number which, in the cases of images $1-10$, is inversely proportional to the extent of the arm misalignment. Figure 4A confirms a trend toward underestimation of the 3D AC-SC image values when the arms were misaligned (3D1-3D9). The extent of this underestimation is seen to become more significant with increasing arm misalignment (decreasing slice number) and increasing arm activity (e.g., 3D1 has greater arm activity than 3D9). 3D10, for which there was no arm

TABLE 1

Scatter Fractions Estimated During Scatter Correction of AC-SC Phantom Images Acquired in 2 and 3 Dimensions

\begin{tabular}{lcc}
\hline Scan no. & 2D scatter fraction & 3D scatter fraction \\
\hline 0 & 0.14 & 0.31 \\
1 & 0.14 & 0.51 \\
2 & 0.14 & 0.50 \\
3 & 0.15 & 0.48 \\
4 & 0.15 & 0.47 \\
5 & 0.16 & 0.46 \\
6 & 0.16 & 0.45 \\
7 & 0.16 & 0.43 \\
8 & 0.17 & 0.42 \\
9 & 0.17 & 0.40 \\
10 & 0.19 & 0.30
\end{tabular}

Scan 0 was acquired with perfect alignment of CT and PET data. All other scans (1-10) were acquired with arms misaligned between PET and CT. 


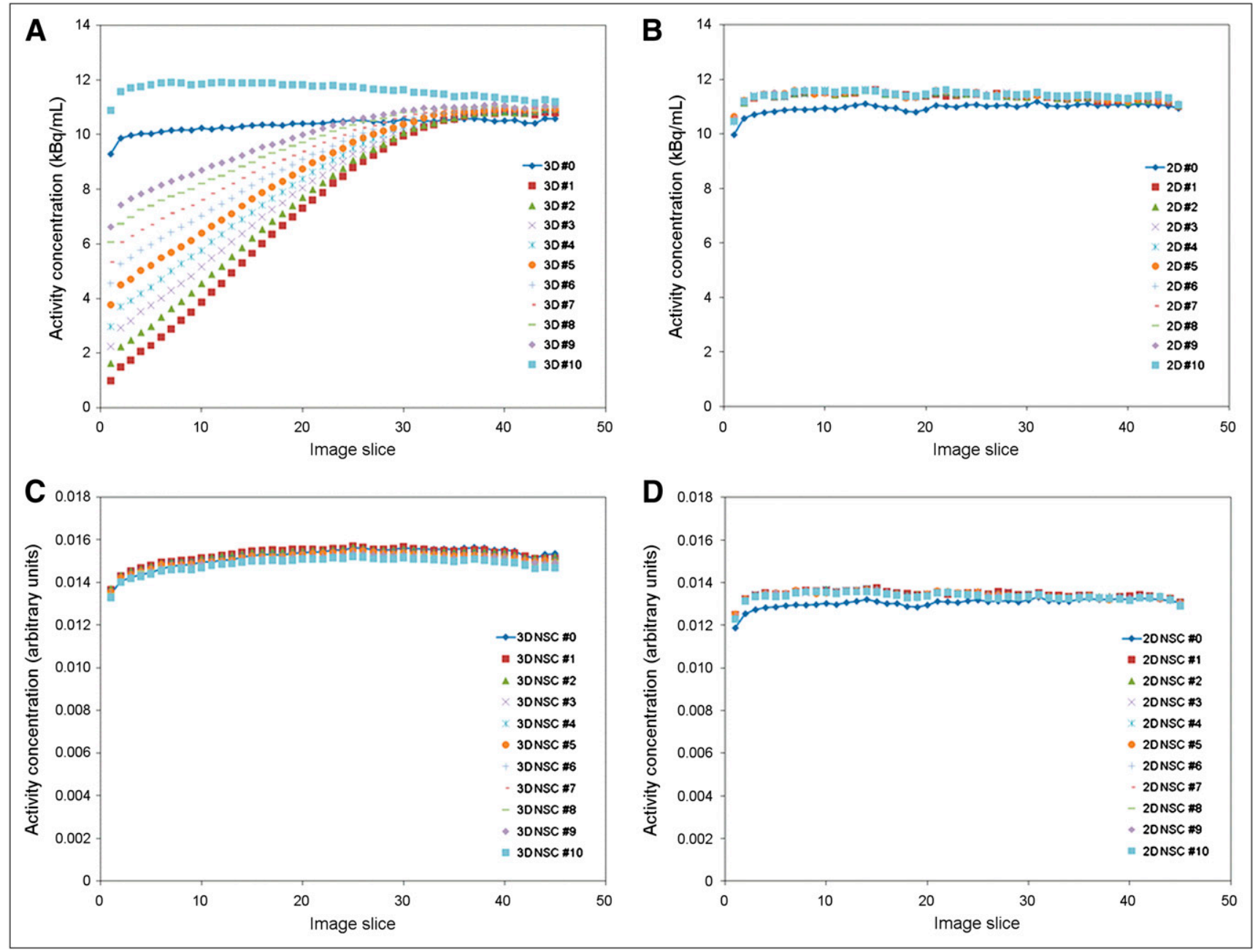

FIGURE 4. Mean activity concentration derived from $19-\mathrm{cm}$-diameter circular region of interest placed inside ${ }^{68} \mathrm{Ge} /{ }^{68} \mathrm{Ga}$ cylinder in reconstructed PET images. Each data series (0 through 10) refers to corresponding image series. 0 corresponds to no misalignment between PET and CT. 1-10 corresponds to misaligned image data. ${ }^{18} \mathrm{~F}$ activity in misaligned arms decreases because of radioactive decay between 1 and 10 such that 10 has no ${ }^{18} \mathrm{~F}$ activity. Data are shown as function of image slice number, which, in image series $1-10$, is inversely related to extent of misalignment between PET and CT. Image slice 45 has approximately no misalignment, whereas image slice 1 has 5-cm misalignment. A and B show fully corrected (AC-SC) data in $3 D$ and $2 D$ modes, respectively. $C$ and $D$ show data with no scatter correction (AC-NSC) in 3D and 2D modes, respectively.

activity, is seen to overestimate the signal relative to 3D0 and is similar to the misaligned 2D data (Fig. 4B, 2D12D10). For these configurations (3D10 and 2D1-2D10), attenuation-correction error, which is expected to be similar in 2D and 3D modes, appears to dominate. Figure 4C further supports the proposition that image reconstruction without scatter correction can be used to suppress the artifacts seen in AC-SC 3D PET. In Figure 4D, the 2D AC-NSC data show trends similar to the $2 \mathrm{D}$ AC-SC data (Fig. 4B), confirming that scatter-correction error is a small effect in 2D.

\section{DISCUSSION}

In this series of clinical observations and experiments, we have tried to characterize and understand the cause of the artifacts associated with patient arm motion during wholebody PET/CT. The dominant artifact manifested as a striking underestimation of the reconstructed image signal throughout multiple transverse slices and appeared as cold bands in the coronal and sagittal planes at the level of the arm motion. In the case of arms-up acquisition, the artifact typically occurred at the neck or shoulder, although it can occur at other sites, as noted in Figure 1J. The artifact was not confined to the specific region in which the arm motion occurred and extended throughout the corresponding transverse slices. Indeed, the effect was greatest at locations deeper within the body, including positions that did not move and were at some distance from the site of the motion. In cases for which these regions are of clinical interest, the presence of the artifact can complicate image interpretation and may require the acquisition of additional data. As the artifacts did not occur in uncorrected (NAC-NSC) PET images, a phantom study was designed to isolate the influence that spatial misalignment had on attenuation and scatter corrections. 
On the basis of these phantom measurements, it became clear that overcorrection for scatter, as opposed to attenuation-correction error, was the cause of the dramatic cold artifacts observed in patient images acquired in 3D. The cold artifact shown in Figure 2G, and quantified in Figure 4A, is seen to decrease in Figures 2H-2J as activity in the arms decayed. Attenuation-correction error cannot explain these changes because the attenuation distribution was identical in each case. Scatter-correction error would seem to provide an explanation because, in the case of both patient and phantom studies, arm motion caused emission activity to appear to be located outside the body, as indicated by CT. 3D scattercorrection algorithms such as the one implemented on the Discovery RX $(20,21)$, and also on other commercial systems $(22,23)$, use data obtained from the tails of the projections to scale the scatter estimate so as to match the patient data. This method assumes that, after randoms correction, coincidence data recorded in the tails of the projections can be attributed to scatter because these projections do not pass through the patient. Identification of the lines of response to use for this scatter estimation is based on the patient's body outline and is obtained from the CT data. In cases of motion, the CT data no longer accurately reflect the position of the patient during PET data acquisition, and this algorithm can break down. The scatter-correction algorithm identifies lines of response that are outside the body at the time of the CT but at the time of the PET acquisition may pass through the body, resulting in a large number of true coincidence events. The correction algorithm, therefore, assumes a high scatter contribution and attempts to compensate by applying a correspondingly high scatter correction. This overcorrection for scatter accounts for the cold artifacts seen in both patient and phantom images.

This explanation is supported by the phantom experiment in several ways, including the relationship between the amount of activity in the arms and the extent of the cold artifact. As activity in the misaligned arms decreased, the number of coincidence events incorrectly assumed to be scatter also decreased, resulting in less extensive overcorrection. When activity in the arms had completely decayed (3D10), no scatter-correction error would have been expected via this mechanism and, as shown in the Figure 2J, no artifacts were observed. At this point, only attenuation-correction error remains and the $2 \mathrm{D}$ and $3 \mathrm{D}$ data are strikingly similar (3D10 and 2D10 in Figs. 4A and 4B, respectively). The fact that the artifact appeared only in the 3D images and not in the $2 \mathrm{D}$ images is also consistent with this explanation. The presence of interplane septa means that $2 \mathrm{D}$ acquisition is associated with much lower scatter fractions than $3 \mathrm{D}$ acquisition and errors in scatter correction would therefore be expected to have a much smaller effect in 2D. Furthermore, the observation that the cold artifact occurred in the 3D images, not at the exact site of the arm motion but toward the center of the scatter distribution, is also consistent with scatter-correction error.

Further evidence that scatter overcorrection causes the cold artifact was provided when image reconstruction without scatter correction (AC-NSC) resulted in complete elimination of the artifact (Figs. 2L-2N). This observation suggests that a potential solution, when artifacts of this kind occur in clinical images, is to repeat image reconstruction without any scatter correction. As seen in the example patient images in Figures $1 \mathrm{~K}-1 \mathrm{O}$, this approach successfully eliminated the cold artifacts and may assist in determining whether patients require repeated imaging. A caveat to this approach is that, although the artifact was eliminated, accurate image quantification requires scatter correction. Indices such as standardized uptake value are, therefore, no longer available.

\section{CONCLUSION}

Reconstruction artifacts due to patient arm motion can be substantial and should be recognized because they can affect both qualitative and quantitative assessment of PET.

\section{DISCLOSURE STATEMENT}

The costs of publication of this article were defrayed in part by the payment of page charges. Therefore, and solely to indicate this fact, this article is hereby marked "advertisement" in accordance with 18 USC section 1734.

\section{ACKNOWLEDGMENT}

No potential conflict of interest relevant to this article was reported.

\section{REFERENCES}

1. Delbeke D, Coleman RE, Guiberteau MJ, et al. Procedure guidelines for tumor imaging with ${ }^{18}$ F-FDG PET/CT 1.0. J Nucl Med. 2006;47:885-895.

2. Boellaard R, O'Doherty MJ, Weber WA, et al. FDG PET and PET/CT: EANM procedure guidelines for tumour PET imaging: version 1.0. Eur J Nucl Med Mol Imaging. 2010;37:181-200.

3. Brooks RA, Di Chiro G. Beam hardening in x-ray reconstructive tomography. Phys Med Biol. 1976;21:390-398.

4. Mawlawi O, Erasmus JJ, Pan T, et al. Truncation artifact on PET/CT: impact on measurements of activity concentration and assessment of a correction algorithm. AJR. 2006;186:1458-1467.

5. Beyer T, Bockisch A, Kuhl H, Martinez MJ. Whole-body ${ }^{18}$ F-FDG PET/CT in the presence of truncation artifacts. J Nucl Med. 2006;47:91-99.

6. Beyer T, Antoch G, Blodgett T, Freudenberg LF, Akhurst T, Mueller S. Dualmodality PET/CT imaging: the effect of respiratory motion on combined image quality in clinical oncology. Eur J Nucl Med Mol Imaging. 2003;30:588-596.

7. Nehmeh SA, Erdi YE. Respiratory motion in positron emission tomography/ computed tomography: a review. Semin Nucl Med. 2008;38:167-176.

8. Teräs M, Kokki T, Durand-Schaefer N, et al. Dual-gated cardiac PET: clinical feasibility study. Eur J Nucl Med Mol Imaging. 2010;37:505-516.

9. Martinez-Möller A, Zikic D, Botnar RM, et al. Dual cardiac-respiratory gated PET: implementation and results from a feasibility study. Eur J Nucl Med Mol Imaging. 2007;34:1447-1454.

10. Lautamäki R, Brown TL, Merrill J, Bengel FM. CT-based attenuation correction in ${ }^{82} \mathrm{Rb}$-myocardial perfusion PET-CT: incidence of misalignment and effect on regional tracer distribution. Eur J Nucl Med Mol Imaging. 2008;35:305-310.

11. Khurshid K, Berger KL, McGough RJ. Automated cardiac motion compensation in PET/CT for accurate reconstruction of PET myocardial perfusion images. Phys Med Biol. 2008;53:5705-5718.

12. Herzog H, Tellmann L, Fulton R, et al. Motion artifact reduction on parametric PET images of neuroreceptor binding. J Nucl Med. 2005;46:1059-1065.

13. Montgomery AJ, Thielmans K, Mehta MA, Turkheimer F, Mustafovic S, Grasby PM. Correction of head movement on PET studies: comparison of methods. J Nucl Med. 2006;47:1936-1944. 
14. Nakamoto Y, Chin BB, Cohade C, Osman M, Tatsumi M, Wahl RL. PET/CT: artifacts caused by bowel motion. Nucl Med Commun. 2004;25:221-225.

15. Lodge MA, Chaudhry MA, Udal DN, Wahl RL. Characterization of a perirectal artifact in ${ }^{18}$ F-FDG PET/CT. J Nucl Med. 2010;51:1501-1506.

16. Kemp BJ, Kim C, Williams JJ, Ganin A, Lowe VJ. NEMA NU 2-2001 performance measurements of an LYSO-based PET/CT system in 2D and 3D acquisition modes. J Nucl Med. 2006;47:1960-1967.

17. Kinahan PE, Townsend DW, Beyer T, Sashin D. Attenuation correction for a combined 3D PET/CT scanner. Med Phys. 1998;25:2046-2053.

18. Ollinger JM. Model-based scatter correction for fully 3D PET. Phys Med Biol. 1996;41:153-176.
19. Bergström M, Eriksson L, Bohm C, Blomqvist G, Litton J. Correction for scattered radiation in a ring detector positron camera by integral transformation of the projections. J Comput Assist Tomogr. 1983;7:42-50.

20. Wollenweber SD. Parameterization of a model-based 3-D PET scatter correction. IEEE Trans Nucl Sci. 2002;49:722-727.

21. Iatrou M, Manjeshwar RM, Ross SG, Thielemans K, Sterans CW. 3D implementation of scatter estimation in 3D PET. IEEE Nucl Sci Symp Conf Rec. 2006: 2142-2145

22. Watson CC, Casey ME, Michel C, Bendriem B. Advances in scatter correction for 3D PET/CT. IEEE Nucl Sci Symp Conf Rec. 2004:3008-3012.

23. Accorsi R, Adam LE, Werner ME, Karp JS. Optimization of a fully $3 \mathrm{D}$ single scatter simulation algorithm for 3D PET. Phys Med Biol. 2004;49:2577-2598. 